\title{
FEATURES OF THE SPERIMAGNETIC STRUCTURE OF TbCO-BASED MULTILAYERS
}

\author{
Makarochkin I.A. ${ }^{1}$, Kudyukov E.V. ${ }^{1}$, Stepanova E.A. ${ }^{1}$, \\ Kurlyandskaya G.V. ${ }^{1}$, Svalov A.V. ${ }^{1}$ \\ 1) Institute of Natural Sciences and Mathematics, Ural Federal University, \\ 620002 Ekaterinburg, Russia \\ E-mail: makarochkin98@,gmail.com
}

The influence of the thickness of the nanoscale ferrimagnetic Tb-Co layers on the magnetic properties of $\mathrm{Tb}-\mathrm{Co} / \mathrm{Ti}$ and $\mathrm{Tb}-\mathrm{Co} / \mathrm{Si}$ multilayers was studied. The fanning cone angle of $\mathrm{Tb}$-Co sperimagnetic structure changes non-monotonously with a decrease of the $\mathrm{Tb}-\mathrm{Co}$ layer thickness.

Tb-Co amorphous thin films and Tb-Co-based magnetic multilayers have attracted special attention due to their high potential in magneto-optical recording and variety of sensor applications $[1,2]$. However, the data related to the thickness dependence of magnetic properties of $\mathrm{Tb}$-Co films are still very limited $[3,4]$. In this work, the influence of the thickness of the nanoscale ferrimagnetic $\mathrm{Tb}$-Co layers on the magnetic properties of $\mathrm{Tb}-\mathrm{Co} / \mathrm{Ti}$ and $\mathrm{Tb}-\mathrm{Co} / \mathrm{Si}$ multilayers was studied.

TbCo-based magnetic multilayers with different thicknesses of Tb-Co layers and non-magnetic spacers ( $\mathrm{Ti}, \mathrm{Si}$ ) have been prepared by rf-sputtering technique. Chemical composition of $\mathrm{Tb}$-Co films deposited from the mosaic target was $\mathrm{Tb}_{27} \mathrm{Co}_{73}$. The magnetic properties of the samples were measured by a SQUID magnetometer.

The descending branches of the hysteresis loop for samples with different thicknesses of $\mathrm{Tb}$-Co layers showed a significant change in the magnetization over the entire range of the used magnetic fields up to $70 \mathrm{kOe}$.

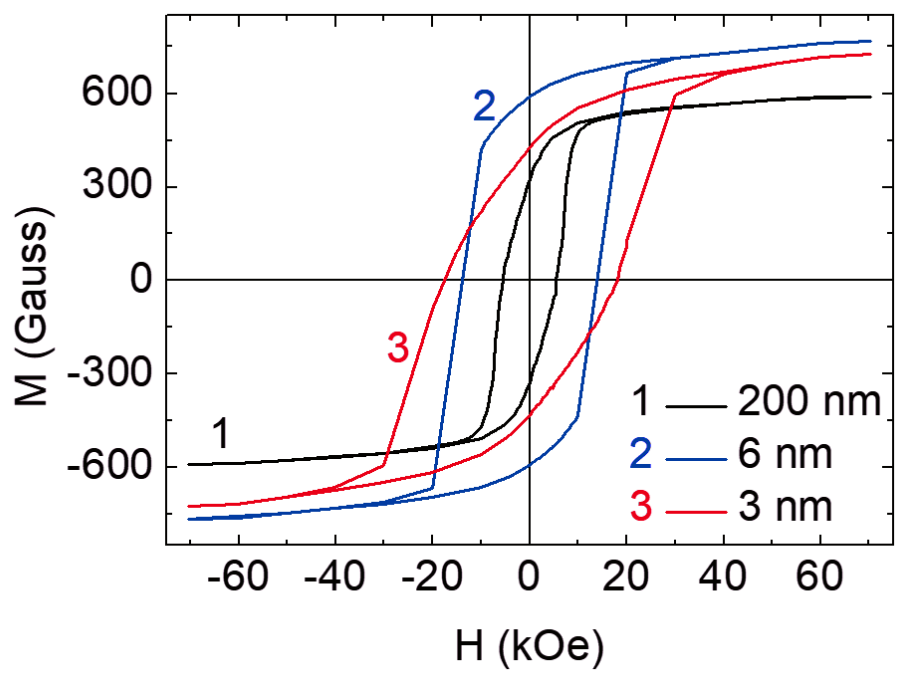

Fig. 1. Hysteresis loops of the Tb-Co(200 nm) film (1), [Tb-Co(6 nm)/Ti(2 nm)]20 (2) and $[\mathrm{Tb}-\mathrm{Co}(3 \mathrm{~nm}) / \mathrm{Ti}(2 \mathrm{~nm})] 30(3)$ multilayers measured at $\mathrm{T}=5 \mathrm{~K}$.

Probably this behavior is a direct consequence of the sperimagnetism of the Tb-Co layers. It can be caused by the distortion of their magnetic structure in response to an 
external magnetic field. In order to determine the effective magnetic moment of terbium atoms and the angular distribution (fanning cone) of the $\mathrm{Tb}$ moments well known method based on the evaluation of the change of the saturation magnetization was used [5]. It was found that the fanning cone angle changes non-monotonously with a decrease of the Tb-Co layer thickness. The paper discusses the reason for this behavior of the fanning cone angle, taking into account a possible reduction of the Co atom moment because of the electron charge transfer from atoms of the non-magnetic spacers to atoms of the transition metal.

This study was supported by RFBR (grant 17-02-00236-a).

1. P.P. Freitas, J.L. Leal, T.S. Plaskett, L.V. Melo, J. C. Soares. J. Appl. Phys. 75 (1994) 6480.

2. M.S. El Hadri, M. Hehn, G. Malinowski, S. Mangin. J. Phys. D: Appl. Phys. 50 (2017) 133002.

3. B. Hebler, A. Hassdenteufel, P. Reinhardt, H. Karl, M. Albrecht. Front. Mater. 3 (2016) 8.

4. A.V. Svalov, E.V. Kudyukov, K.G. Balymov, E.A. Stepanova, V.O. Vas'kovskiy, A. Larrañaga, G.V. Kurlyandskaya. Phys. Met. Metallogr. 120 (2019) 18.

5. V.O. Vas'kovskiy, O.A. Adanakova, K.G. Balymov, N.A. Kulesh, A.V. Svalov, E.A. Stepanova. Phys. Solid State 57 (2015) 1142. 